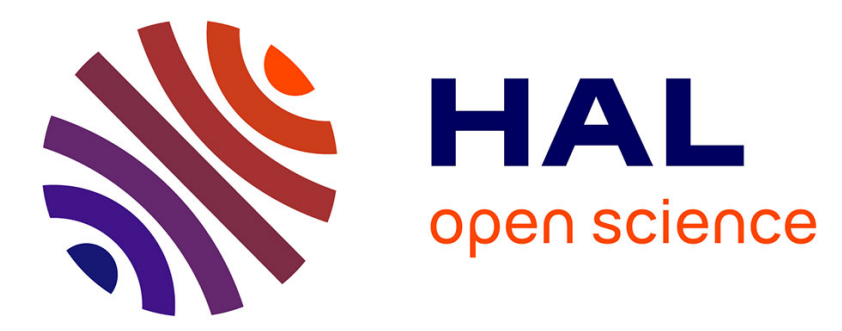

\title{
Time-resolved buildup of a photorefractive grating induced in Bi12SiO20 by picosecond light pulses
}

\author{
Jean-Michel Jonathan, Gérald Roosen, Philippe Roussignol
}

\section{To cite this version:}

Jean-Michel Jonathan, Gérald Roosen, Philippe Roussignol. Time-resolved buildup of a photorefractive grating induced in Bi12SiO20 by picosecond light pulses. Optics Letters, 1988, 13 (3), pp.224-226. 10.1364/OL.13.000224 . hal-00877677

\section{HAL Id: hal-00877677 \\ https://hal-iogs.archives-ouvertes.fr/hal-00877677}

Submitted on 29 Oct 2013

HAL is a multi-disciplinary open access archive for the deposit and dissemination of scientific research documents, whether they are published or not. The documents may come from teaching and research institutions in France or abroad, or from public or private research centers.
L'archive ouverte pluridisciplinaire HAL, est destinée au dépôt et à la diffusion de documents scientifiques de niveau recherche, publiés ou non, émanant des établissements d'enseignement et de recherche français ou étrangers, des laboratoires publics ou privés. 


\title{
Time-resolved buildup of a photorefractive grating induced in $\mathrm{Bi}_{12} \mathrm{SiO}_{20}$ by picosecond light pulses
}

\author{
J. M. C. Jonathan and G. Roosen \\ Institut d'Optique Théorique et Appliquée, U.A. au Centre National de la Recherche Scientifique No. 14, Centre Universitaire d'Orsay, \\ Bâtiment 503, B.P. 43, 91406 Orsay Cedex, France

\section{Ph. Roussignol} \\ Laboratoire d'Optique Quantique du Centre National de la Recherche Scientifique Ecole Polytechnique, Route de Saclay, 91120 Palaiseau,
} France

Received October 15, 1987; accepted December 23, 1987

\begin{abstract}
Photorefractive gratings are induced with picosecond light pulses in a BSO crystal. Both experiment and calculations show a buildup of the effect governed by a diffusion of the excited charge carriers that occurs after illumination.
\end{abstract}

The photorefractive effect, identified in many insulator and semiconductor crystals, has permitted the demonstration of a wide variety of operations in the fields of phase conjugation and optical signal processing. ${ }^{1-5}$ It results from a light-induced charge redistribution in the crystalline material that generates a space-charge electrostatic field. In turn the refractive index of the material is modulated through the electro-optic effect. ${ }^{6}$ As a consequence, the photorefractive effect is essentially sensitive to the absorbed optical energy. Experiments conducted in various laboratories have shown that the magnitude of the photoinduced space-charge field increases with energy. ${ }^{7-9}$ However, studies performed with nanosecond light pulses (in $\mathrm{BSO}, \mathrm{BaTiO}_{3}$, and $\mathrm{LiNbO}_{3}$ ) as well as with picosecond light excitations ${ }^{10,11}$ (in $\mathrm{GaAs}$ and $\mathrm{BaTiO}_{3}$ ) did not show evidence of the ultimate speed of the photorefractive effect; the grating was always completed within the pulse duration $\tau_{L}$.

From the band-transport model used for the photorefractive effect ${ }^{6}$ one derives the ratio of the diffusion time $\left(\tau_{d}\right)$ to the recombination time $\left(\tau_{R}\right)$ of the charge carriers:

$$
\tau_{d} / \tau_{R}=(\Lambda / 2 \pi L)^{2},
$$

where $L$ is the diffusion length of the photoexcited charge carriers and $\Lambda$ is the spacing of the induced grating. Measurements of $L$ in various sillenite crystals $^{12}$ indicate that $\tau_{d}$ is easily made smaller than $\tau_{R}$. Efficient recording of a photorefractive grating in BSO crystals with light pulses of duration much shorter than the diffusion time is thus possible. ${ }^{13}$ In this Letter we present the first reported demonstration of such behavior. Moreover, analysis of the results gives an estimate of the diffusion time of the excited charge carriers and consequently of their mobility.

The experimental arrangement is shown in Fig. 1. The laser source is a mode-locked Nd:YAG laser from which single pulses are extracted, using a Pockels cell, and then amplified. After frequency doubling, the average pulse width is $28 \mathrm{psec}$ and its energy is $0.5 \mathrm{~mJ}$. The repetition rate is $2 \mathrm{~Hz}$. The beam is split into three parts by beam splitters BS1 and BS2. Beams $\mathrm{S}_{1}$ and $R$ are counterpropagating, and $S_{2}$ makes an angle of $13^{\circ}$ with $S_{1}$. $S_{1}$ and $S_{2}$ may thus record a $2.3-\mu \mathrm{m}$ spacing grating, while $S_{2}$ and $R$ may record a $0.3-\mu \mathrm{m}$ period grating in the BSO crystal. The energy of pulse $S_{2}$ is approximately $1 \%$ of that of $S_{1}$ and $R$. Two adjustable delay lines permit a delay on any of the three pulses. The conjugated signal $S$, extracted by

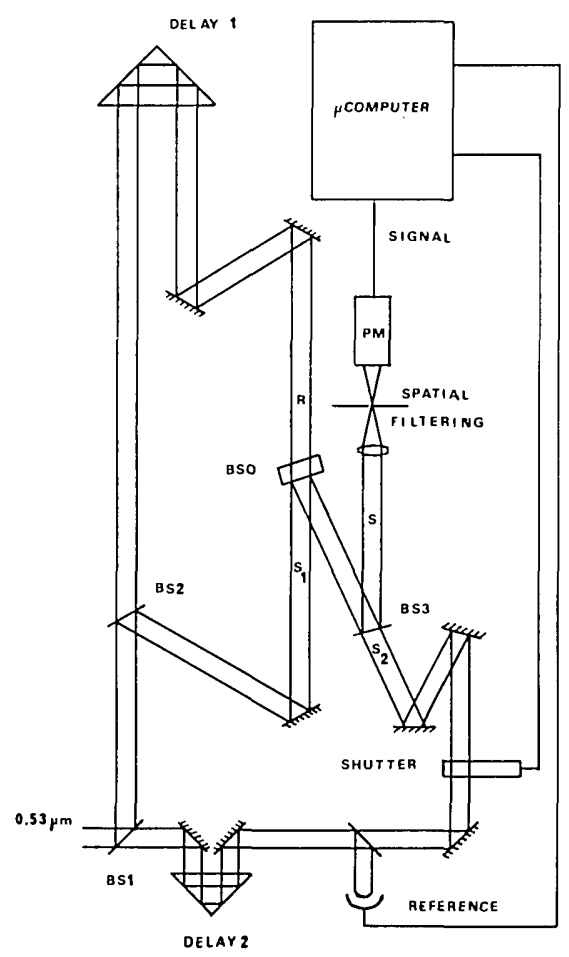

Fig. 1. Experimental arrangement for the time-resolved study of the photorefractive effect. PM, photomultiplier. 


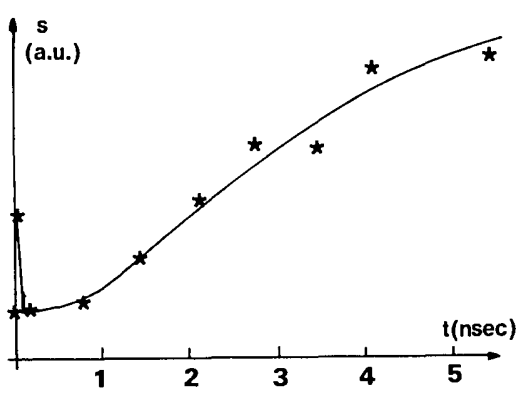

Fig. 2. Time evolution of the diffraction efficiency.

beam splitter BS3, is detected by a photomultiplier tube (PMT) after spatial filtering. The signal is compared with a reference signal from a P-I-N photodiode.

To minimize beam-coupling effects, the BSO crystal is oriented with its crystallographic planes (001) and (i10), respectively, parallel to the plane of incidence and perpendicular to the grating wave vector.

The analysis of the temporal evolution of a photorefractive grating resulting from the interference of two single pulses (say, $S_{1}$ and $S_{2}$ ) is conducted as follows. First beam $S_{2}$ is blocked by a shutter. $S_{1}$ and $R$ thus erase any prerecorded grating. When the magnitude of the signal from the PMT is below a given threshold corresponding to background light, the shutter is opened and single pulses $S_{1}$ and $S_{2}$ interfere in the $\mathrm{BSO}$ crystal. The strength of the photoinduced index grating is probed by a single pulse $\mathrm{R}$ that can be delayed from 0 to $5 \mathrm{nsec}$. The signal from the PMT is stored in the microcomputer, and the shutter is closed to achieve the erasure step before another measurement.

For each value of the delay on $\mathrm{R}$, the diffracted signal is recorded and averaged over 20 measurements. No electric field was applied to the crystal. Figure 2 shows a plot of the results.

Two distinct features are visible on this recording. First comes a sharp peak, the width of which is similar to that of the intensity autocorrelation function of the light pulses. Thus a phase-conjugate beam is generated as the three input pulses are coincident in the BSO crystal. Such a signal was previously seen in BSO (Ref. 14) and is attributed to the third-order nonlinear susceptibility. We found that the BSO phase-conjugate reflectivity is about 30 times smaller than in $\mathrm{CS}_{2}$ in the same experimental conditions.

The second part of the recording shows a buildup of a grating well after the illumination end; its time constant is approximately $4 \mathrm{nsec}$. This grating exists only when the polarizations of beams $S_{1}$ and $S_{2}$ are parallel, and it persists in the darklike photorefractive gratings recorded with quasi-continuous light excitations. This has been verified by measuring the grating efficiency with a pulse emitted by the laser a few seconds after the writing pulses. Such persistence in the dark is a good indication of a photorefractive grating. Establishment of the effect far after the interfering pulses does not permit two-beam coupling experiments, which are unequivocal evidence of the photorefractive effect.
In this experiment the diffraction efficiency was approximately $5 \times 10^{-8}$. This low value is not to be attributed to an erasure by the reading pulse, as the building rate is much larger than the pulse duration. It is related to the low energy of the writing pulses. No diffracted signal could be observed when $S_{1}$ was delayed relative to $S_{2}$ and $R$. Thus the magnitude of the small-spacing $(0.3-\mu \mathrm{m})$ photoinduced grating should be much smaller than that of the larger one (2.3 $\mu \mathrm{m})$. This point is confirmed by theoretical analysis.

The buildup of the photorefractive grating may be described using the basic band-iransport model ${ }^{6}$ with a single kind of charge carrier and a single species of photorefractive trap. The basic equations (rate, continuity, and current equations and Poisson's law) are linearized, using the assumption that the modulation of the illuminating fringes is small enough to ensure sinusoidal variation of any spatially varying functions in the equations. No other approximation was made. A numerical integration of the material differential equations is performed using our experimental data: cw measurements of the diffusion length ${ }^{12}(L=3.4$ $\mu \mathrm{m}$ ) in this crystal lead to $\tau_{d} / \tau_{R}=0.01$; no photorefractive effect is observed during the 28-psec pulse duration.

Pulse duration and temporal shape, chosen as Gaussian, appear to be of little importance in the computation. Results of the numerical calculation are shown in Fig. 3 for our experimental conditions (pulse duration much shorter than recombination time, modulation of the illumination grating $m=0.2$ ). The time is expressed in units of $\tau_{R}$ and the charge density in units of $N_{A}$ (trap density), and we used a normalized intensity $\bar{I}_{0}\left(I_{0}=s I_{0} \tau_{R}\right)$ corresponding to our experimental conditions. The photoexcitation cross section $s$ was estimated to be $10^{-5} \mathrm{~m}^{2} \mathrm{~J}^{-1} .15$ Figure 3(a) shows the diffraction-efficiency buildup reaching its steady
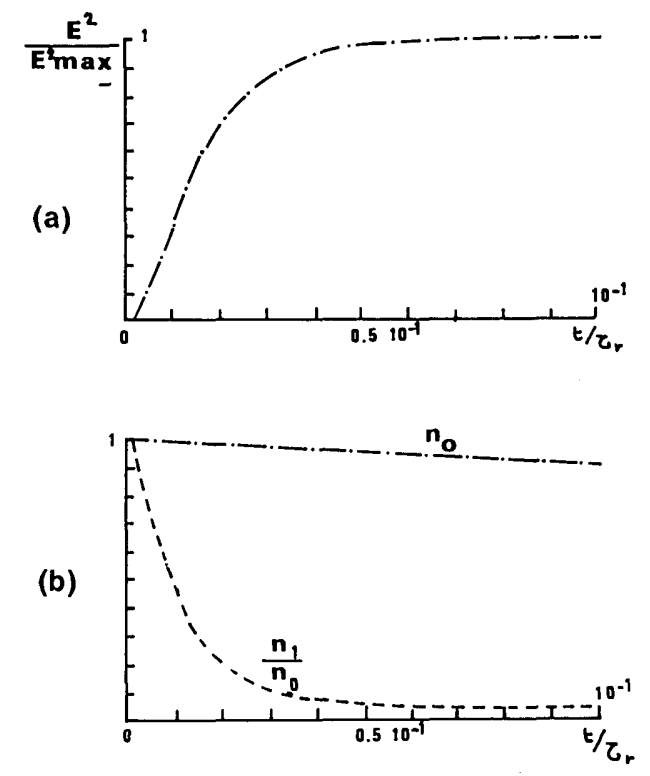

Fig. 3. Computer simulation of the buildup: (a) normalized square of the space-charge field (proportional to the diffraction efficiency); (b) normalized uniform density of free carriers $\left(n_{0} / N_{A}\right)$ and spatial modulation of the free carriers $\left(n_{1} / n_{0}\right)$. 


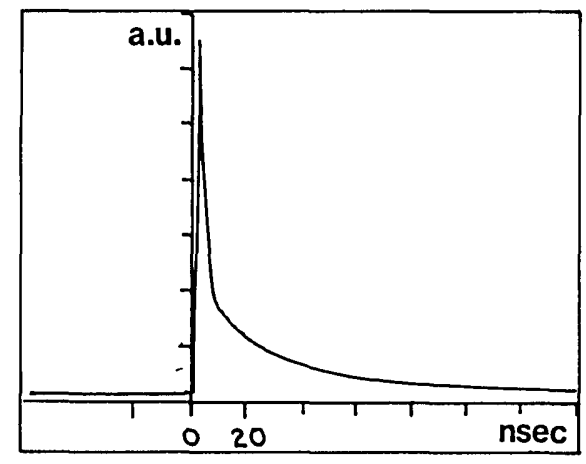

Fig. 4. Experimental decay of the photocurrent (redrawn from an oscilloscope trace; resolution $2 \mathrm{nsec}$ ).

state at $t \simeq 0.04 \tau_{R}$. The processes involved are illustrated by the time evolution of the excited charge densities [Fig. 3(b)]. The dc part of the carrier density, $n_{0}$, remains constant, while its spatial modulation, $n_{1} / n_{0}$, decreases when diffraction efficiency increases. There is little carrier recombination but only a diffusion process leading to a difference in the respective modulations of ion and excited charge densities. This induces the space-charge field. The steady state is obtained when the diffusion process is balanced by the effect of the induced space-charge field on the carriers. Carrier recombination takes place well after the effect and has no effect on it.

The steady-state amplitude first increases proportionally to the optical energy while the speed of the effect remains constant. At higher energies a saturation occurs. The space-charge field amplitude, calculated using cur experimental data, is $\simeq 3 \mathrm{~V} / \mathrm{cm}$, in agreement with the observed diffraction efficiency.

For the smaller-period grating, $\left(\tau_{d} / \tau_{R}=10^{-4}\right)$, the calculations predict an extremely fast buildup, in the picosecond range, with a much lower amplitude of the induced space-charge field. This would lead to a diffracted signal 2 orders of magnitude smaller than the one produced by the large-spacing grating. This signal could not be detected in our experiment.

For the $2.3-\mu \mathrm{m}$-period grating the numerical calculation predicts a buildup time equal to $4 \%$ of the recombination time $\tau_{R}$ [Fig. 3(a)], while experimental results give a value of $4 \mathrm{nsec}$ (Fig. 2). Thus the recombination time of the charge carriers in this BSO sample may be estimated roughly as 100 nsec. Since the diffusion time $\tau_{d}$ is $1 \%$ of $\tau_{R}$, the mobility of the excited charge carriers, i.e., electrons, ${ }^{16}$ is

$$
\mu=\frac{e \Lambda^{2}}{k_{B} T 4 \pi^{2} \tau_{d}} \simeq 50 \mathrm{~cm}^{2} \mathrm{~V}^{-1} \mathrm{sec}^{-1},
$$

where $e$ is the electric charge, $k_{B}$ the Boltzmann constant, and $T$ the absolute temperature.

We tried to find this recombination time by looking at the time behavior of the photocurrent generated by a 28-psec light pulse. Resolution was limited to $1 \mathrm{nsec}$ by the detector and the oscilloscope. The results (Fig. 4) show a fast decay of the photocurrent (smaller than 1 nsec) followed by a slower one. The analysis reveals a multiple exponential decay with time constants ranging from $30 \mathrm{nsec}$ to $12 \mu \mathrm{sec}$. This could be attributed to a complex impurity level structure, which is not taken into account in the model. However, the experimental results suggest that additional trapping processes faster than $100 \mathrm{nsec}$ would not influence the speed of the space-charge field buildup.

In conclusion, a space-charge electrostatic field has been generated by pure diffusion of the excited charge carriers. The field results from the differences in the modulations of the spatial distributions of ions and free charges. This is made possible, in materials such as BSO, by the materials' large diffusion length. Similar behavior should be exhibited by semiconductors such as GaAs. Much higher carrier mobility in those materials should contribute to an even shorter buildup time. In ferroelectric crystals, however, $\tau_{d} \gg \tau_{R}$, because of the small value of the diffusion length. Therefore space-charge buildup must be governed by both recombination and diffusion. Consequently a photorefractive grating may be induced only by light pulses whose duration $\tau_{L}$ is near the diffusion time, i.e., $\tau_{L} \gg \tau_{R}$.

The authors thank D. Ricard for valuable comments.

\section{References}

1. P. Günter, Phys. Rep. 93, 199 (1982).

2. S. I. Stepanov and M. P. Petrov, Opt. Acta 31, 1335 (1984).

3. M. Cronin-Golomb, B. Fisher, J. O. White, and A. Yariv, IEEE J. Quantum Electron. QE-20, 12 (1984).

4. J. P. Huignard, H. Rajbenbach, Ph. Refregier, and L. Solymar, Opt. Eng. 24, 586 (1985).

5. J. P. Huignard and G. Roosen, in Nonlinear Optics: Materials and Devices, C. Flytzanis and J. L. Oudar, eds., Vol. 7 of Proceedings in Physics (Springer-Verlag, Berlin, 1985), p. 128.

6. N. V. Kukhtarev, V. B. Markov, S. G. Odulov, M. S. Soskin, and V. L. Vinetskii, Ferroelectrics 22, 949 (1979).

7. C. T. Chen, D. M. Kim, and D. Von der Linde, IEEE J. Quantum Electron. QE-16, 126 (1980).

8. L. K. Lam, T. Y. Chang, J. Feinberg, and R. W. Hellwarth, Opt. Lett. 6, 475 (1981).

9. G. Le Saux, G. Roosen, and A. Brun, Opt. Commun. 56, 374 (1986); 58, 238 (1986).

10. G. C. Valley, A. L. Smirl, M. B. Klein, K. Bohnert, and T. F. Boggess, Opt. Lett. 11, 647 (1986).

11. A. L. Smirl, G. C. Valley, K. Bohnert, and T. F. Boggess, in Digest of Topical Meeting on Photorefractive Materials, Effects and Devices (Optical Society of America, Washington, D.C., 1987), p. 88.

12. G. Pauliat, J. M. C. Jonathan, M. Allain, J. C. Launay, and G. Roosen, Opt. Commun. 59, 266 (1986).

13. J. M. C. Jonathan, Ph. Roussignol, and G. Roosen, in Digest of Topical Meeting on Photorefractive Materials, Effects and Devices (Optical Society of America, Washington, D.C., 1987), p. 97.

14. J. L. Ferrier, J. Gazengel, X. Nguyen Phu, and G. Rivoire, Opt. Commun. 58, 343 (1986).

15. M. Peltier and F. Micheron, J. Appl. Phys. 48, 3683 (1977).

16. G. Pauliat, M. Allain, J. C. Launay, and G. Roosen, Opt. Commun. 61, 321 (1987). 